\title{
A P-band GaN Power Amplifier with LRC-LC Stability Network
}

\author{
Xinyu $\mathrm{Ma}{ }^{\mathrm{a}}$, Baoxing Duan ${ }^{\mathrm{b}}$, and Yintang Yang \\ School of Microelectronics, Xidian University, Xian 710071, China. \\ amaxinyu1993@126.com bbxduan@163.com
}

Keywords: P-band, GaN, stability network, power amplifier, high efficiency

\begin{abstract}
A P-band high-efficiency, high power GaN power amplifier is designed and relized on the basis of the push-pull structure. The LRC-LC stability network is proposed and applied to the power amplifier circuit for the first time. The LRC-LC stability network can significantly reduce the high gain out the band, which eliminate the instability of the power amplifier circuit. The developed power amplifier exhibits $58.5 \mathrm{dBm}(700 \mathrm{~W})$ power output with a $17 \mathrm{~dB}$ gain and $85 \% \mathrm{PAE}$ at $500-600 \mathrm{MHz}$, $300 u s, 20 \%$ duty cycle. It has the highest PAE in P-band among the products at home and abroad.
\end{abstract}

\section{Introduction}

With the rapid development of the modern radio systems, communications equipment, radar performance has achieved unprecedented progress. As one of the most important modules, power amplifier directly affects the performance of radio system [1]. Power devices based on $\mathrm{Si}, \mathrm{GaAs}$ or other materials are limited by gain, bandwidth, thermal stability, and output power density. Gradually unable to meet the requirements of equipments for the high power and high efficiency [2].

In recent years, the third generation of semiconductor materials, such as the $\mathrm{GaN}, \mathrm{SiC}$, which has higher electron mobility and high thermal conduction efficiency. So it can work under high voltage conditions and achieve high power and wide bandwidth in a smaller volume. When the GaN material used in the production of power amplifier, it is necessary to meet the requirements about gain, efficiency, power, flatness and others, and the most important thing is stability. GaN HEMT has a higher power density, so it is more prone to instability at low frequency ${ }^{[3]}$.

To eliminate the instability of GaN power amplifier at low frequency, the LRC-LC stability network structure is proposed. By connecting the stability network in front of the input matching network. The stability of the power amplifier is significantly improved by reducing the gain in low frequency band.

\section{LRC-LC stability network}

\subsection{LRC-LC structure}

In order to improve the stability of low frequency, high power power amplifier, commonly used methods are the followings. For example, putting some consuming components such as resistors in series or parallel in front of the input circuit; putting RC network parallelled to the ground or adding the negative feedback. These methods stabilize the amplifier, but meanwhile, the consuming components will reduce the gain of power amplifier, which affects the efficiency of power amplifier.

The LRC-LC stability network is proposed in this paper, as shown in Figure 1 . The concept of the filter is applied to the design of power amplifier, which significantly improves the stability of the power amplifier without affecting the gain and efficiency.

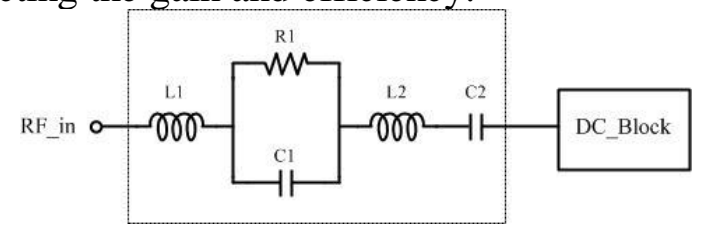

Fig. 1 Schematic of LRC-LC 


\subsection{Simulation of LRC-LC Structure}

The S11 curve is obtained by simulating the circuit in Figure 1, as is shown in Figure 2. It can be seen that this stability network is well-behaved between $500-600 \mathrm{MHz}$ and does not affect the standing wave of the power amplifier circuit.

The S21 curve is compared between the LRC-LC structure that proposed in this paper and traditional RC stability structure, as is shown in Figure 3. It can be seen that the LRC-LC stability network increases the insertion loss out of band, compared with the traditional RC stability structure. Which means the LRC-LC is more effective in improving stability.

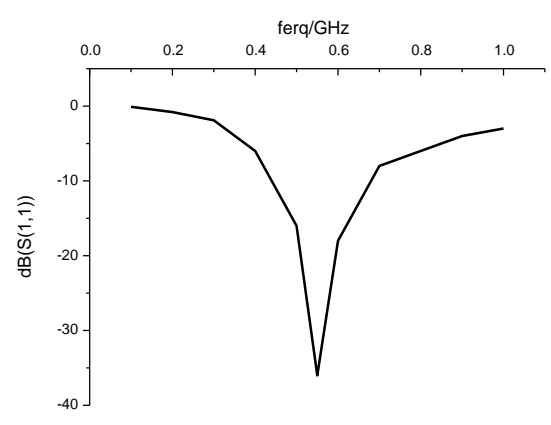

Fig. 2 S11 of LRC-LC

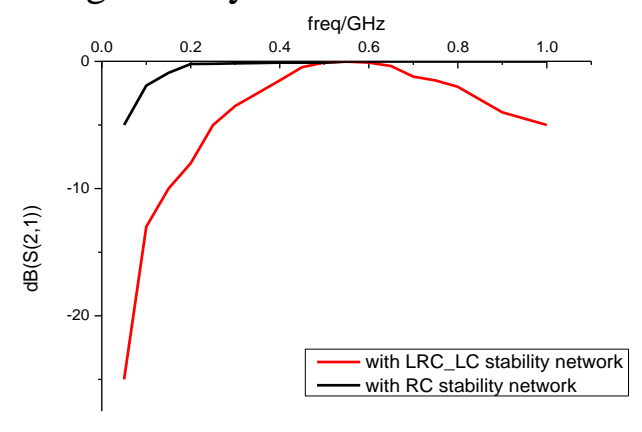

Fig. 3 S21 of LRC-LC and RC

Putting the LRC-LC stability network into the power amplifier circuit. Simulation software is used to simulate the gain and stability of the power amplifier circuit. And the simulation results are compared with the power amplifiers without the stability network. The results are shown in Figure 4 and Figure 5. It can be seen that the gain in low frequency is significantly inhibited, and the stability of the power amplifier circuit is significantly improved with the using of LRC-LC stability network. Eliminating the instability of GaN power amplifier caused by the high gain in low frequency.

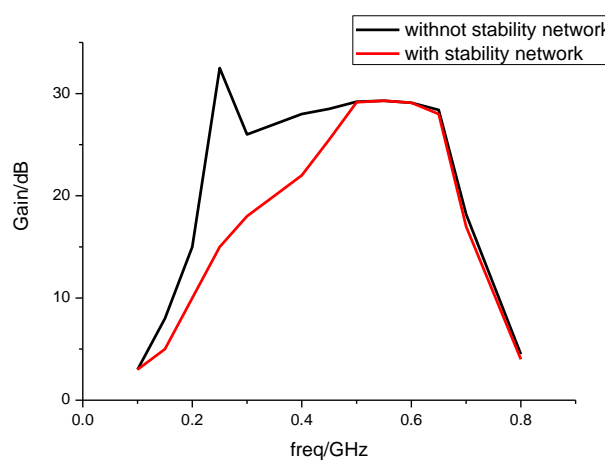

Fig. 4 Gain of power amplifier

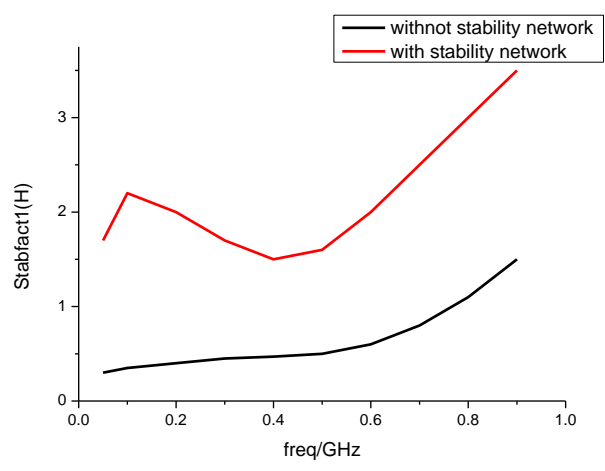

Fig. 5 Stabfact1 of power amplifier

\section{Power amplifier circuit design}

\subsection{Circuit inside tube}

When designing a power amplifier, the total gate width of power chips have a very significant impact on output power and efficiency of the power amplifier ${ }^{[4]}$. Limited to the output capacity of signal unit cell, Parallel connections are used to increase the total gate width, Thereby obtaining the corresponding output power. Considering the impedance of high-power GaN power amplifier is low. In order to reduce the difficulty of matching circuit design, the L-C-L matching method is used in the tube to improve the device's input impedance ${ }^{[5]}$. The equivalent circuit is shown in Figure 6.

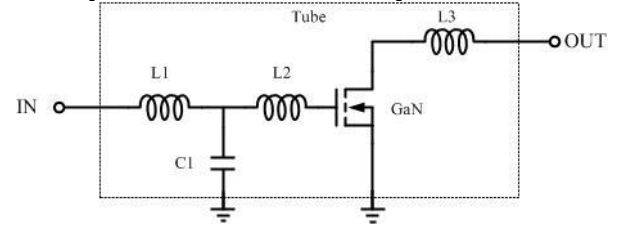

Fig.6 Schematic of power amplifier pre-matching circuit 


\subsection{Matching circuit design}

In the designing of the input and output matching circuits of power amplifier, the input circuit mainly affects stability, insertion loss and gain flatness; the output circuit mainly affects output power and efficiency. First, the input and output circuits are built and simulated by ADS software, and then the amplifier is tested and debugged. Eventually get the required power amplifier.

Traditional push-pull power amplifier circuit is optimized by adding the LRC-LC stability network. The schematic of circuit is shown in Figure 7.

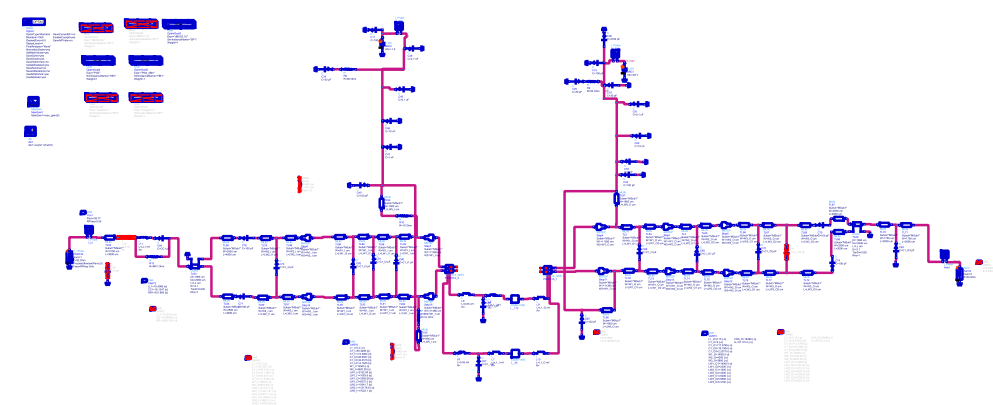

Fig.7 Schematic of circuit

The signal produces a 180 degree phase difference through the balun structure at the input circuit, and respectively amplified by the power dies, then gets a same phase and synthesized by the balun structure at the output circuit.

Balun structure can increase bandwidth, gain and have helped to amplifies power and harmonic suppression in signal transmission ${ }^{[6]}$. The push-pull structure with LRC-LC stability network achieves flat power gain, better matching characteristics and high stability. Therefore, the push-pull balanced structure with a LRC-LC stability network can ensure good stability and increase the amplifier's power gain and bandwidth ${ }^{[7]}$.

\section{Circuit simulation and testing}

\subsection{Circuit simulation}

Electromagnetic Field Simulation is used in the simulation of power die, tube, bonding wire, single layer capacitor and other passive components to improve the accuracy of simulation ${ }^{[8]}$. The simulation of the schematic does not take into account of the coupling among the devices, so there will be a certain gap compared with the actual circuit. So the simulation of circuit layout is necessary to improve the accuracy.

After debugging and optimization, the schematic of the circuit is completely replaced by the layout. After simulation with ADS software, results are as follows: S11 is less than -15dB, the output power is greater than $58.5 \mathrm{dBm}(700 \mathrm{~W})$, the PAE is greater than $80 \%$, as shown in Figure 8 and Figure 9. While the flatness is less than $0.3 \mathrm{~dB}$ and the gain is $17 \mathrm{~dB}$ with the power amplifier working in $500-600 \mathrm{MHz}$. Showing an excellent performance.

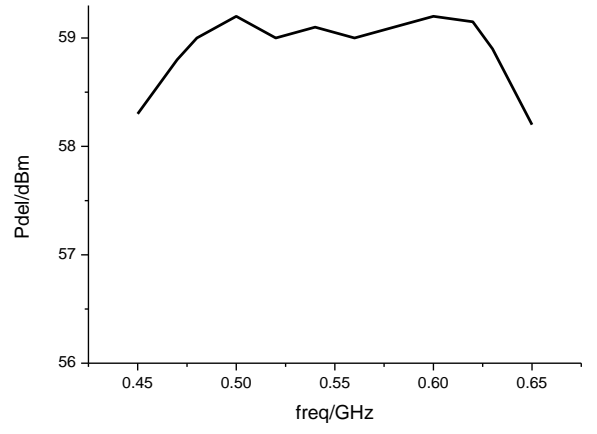

Fig.8 Schematic of output power

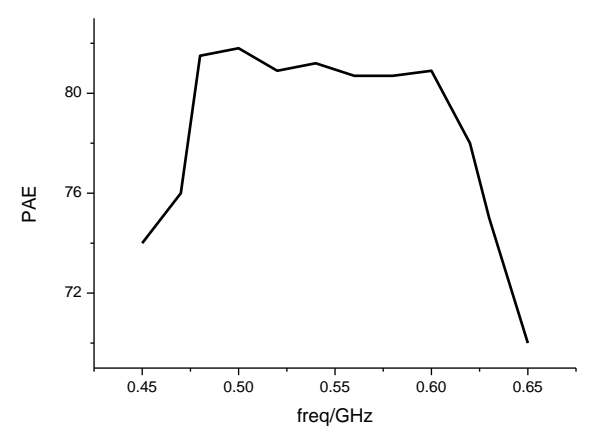

Fig.9 Schematic of PAE 


\subsection{Power amplifier testing}

After ADS simulation, the circuit is produced. The total size of this power amplifier circuit is $110 \times 60 \mathrm{~mm}^{2}$, as shown in Figure 10. RF circuit testing system (signal source, power supply, microwave power sensor, attenuator) is used to test this RF power amplifier. In order to achieve a good impedance matching with the testing system, RF connector is welded to a $4 \mathrm{~mm}$ long $50 \Omega$ microstrip line. Setting the power amplifier bias condition: $\mathrm{Vd}=48 \mathrm{~V}, \mathrm{Vg}=-2.3 \mathrm{~V}$, input signal is 300us, $20 \%$ duty, Pin=41.5dBm. The test results between $500-600 \mathrm{MHz}$, are as follows: Gain $>17 \mathrm{~dB}$, Pout $>58.5 \mathrm{dBm}(700 \mathrm{~W})$ with a maximum Pout of $58.8 \mathrm{dBm}(760 \mathrm{~W})$, PAE $>85 \%$ with a maximum PAE of $87 \%$.

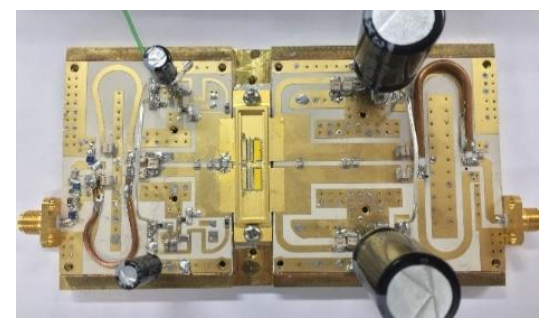

Fig.10 Photo of power amplifier

\section{Conclusion}

A method is proposed to improve the stability of high-power, low-frequency power amplifier, that is, put the LRC-LC stability network into the input circuit of the RF power amplifier. The LRC-LC stability network reduces high gain out the band without affecting the bandwidth and the gain inside the band, which significantly improve the stability of the power amplifier circuit. The developed power amplifier exhibits $58.5 \mathrm{dBm}(700 \mathrm{~W})$ power output with a $17 \mathrm{~dB}$ gain and $85 \%$ PAE at $500-600 \mathrm{MHz}$, 300 us, $20 \%$ duty cycle. It has the highest PAE in P-band among the products at home and abroad.

\section{References}

[1] Wang Jiazhong. Development trend of wireless communication power amplifier , Electronic Engineering \& Product World,2004.1,P35-39

[2] Zeng Yun. The basic of microelectronic devices [M] . Changsha. : Hunan UniversityPress, 2005 .

[3] Liang Xiaofang. Stabity analysis and design of $\mathrm{x}$-band solid-state power amplifier [J].Modern Radar, 2007,29(12):98-100.

[4] Yao Xiaojiang,Li Bin .AlGaN/GaN HEMTs Power Amplifier MIC with Power Combining at C-Band.Chinese Journal of Semiconductors,2007,28(4).

[5] Hirota T. Tarusawa Y. Ogawa H. Uniplanar MMIC Hybrids-a proposed new MMIC structure. IEEE Transactions on Microwave Theory and Techniques, 1987, 35: 576-581

[6] TASKER PJ, Benedikt J. Waveform Inspired Models and the Harmonic Balance Emulator[J]. IEEE Microwave Magazine, 2011, 12(2): 38-54.

[7] Hao Mingli, Liu Xunchun. Method for eliminating low frequency oscillation in $X$ band low noise amplifier [J].Chinese Journal of Electron Devices, 2007,30(2):507-509

[8] K.Krishnamurthy,J.Martin,B.Landberg,R.Vetury,M.J.Poulton.Wideband 400 W Pulsed Power GaN HEMT Amplifiers.Microelectronics 2008 IEEE,2008,3(1):303-306. 